\title{
Association of Plasma Biomarkers for Angiogenesis and Proteinopathy in Indian Amyotrophic Lateral Sclerosis Patients
}

\author{
Shweta Modgil ${ }^{1}$ Radhika Khosla ${ }^{1}$ Abha Tiwari ${ }^{1} \quad K^{2}$ aushal Sharma ${ }^{1}$ Akshay Anand ${ }^{1}$ \\ ${ }^{1}$ Department of Neurology, Neuroscience Research Lab, \\ Postgraduate Institute of Medical Education and Research, \\ Chandigarh, India

\begin{abstract}
Address for correspondence Akshay Anand, PhD, Department of Neurology, Neuroscience Research Lab, Postgraduate Institute of Medical Education and Research, Chandigarh, India 160012 (e-mail: akshay1anand@rediffmail.com).
\end{abstract}

J Neurosci Rural Pract 2020;11:573-580

DOI https://doi.org/ $10.1055 / \mathrm{s}-0040-1714314$ ISSN 0976-3147.
Background Amyotrophic lateral sclerosis (ALS) is a rare motor neuron disease with progressive degeneration of motor neurons. Various molecules have been explored to provide the early diagnostic/prognostic tool for ALS without getting much success in the field and miscellaneous reports studied in various population.

Objective The study was aimed to see the differential expression of proteins involved in angiogenesis (angiogenin [ANG], vascular endothelial growth factor [VEGF], vascular endothelial growth factor receptor 2 [VEGFR2], etc), proteinopathy (transactive response DNA binding protein-43 [TDP-43] and optineurin [OPTN]), and neuroinflammation (monocyte chemoattractant protein-1[MCP-1]) based on the characteristics of ALS pathology. Though, suitable panel based on protein expression profile can be designed to robust the ALS identification by enhancing the prognostic and diagnostic efficacy for ALS.

Methods A total of 89 ALS patients and 98 nonneurological controls were analyzed for the protein expression. Expression of angiogenic (VEGF, VEGFR2, and ANG), neuroinflammation (MCP-1), and proteinopathy (TDP-43 and OPTN) markers were estimated in plasma of the participants. Proteins were normalized with respective value of total protein before employing statistical analysis.

Results Analysis has exhibited significantly reduced expression of angiogenic, proteinopathy, and neuroinflammation biomarkers in ALS patients in comparison to controls. Spearman's correlation analysis has showed the positive correlation to each protein.

Conclusion Altered expression of these proteins is indicating the prominent function in ALS pathology which may be interdependent and may have a synergistic role. Hence, a panel of expression can be proposed to diagnose ALS patient which may also suggest the modulation of therapeutic strategy according to expression profile of patient.

- optineurin

- MCP-1/CCL-2

(C)2020 Association for Helping Neurosurgical Sick People
License terms

() (1) $\ominus \circledast$ 


\section{Introduction}

Amyotrophic lateral sclerosis (ALS), also known as Lou Gehrig's disease, is a devastating neurodegenerative disease characterized by progressive degeneration of neurons and muscles. ${ }^{1}$ The disease is believed to share common genetic link; however, only $10 \%$ of the diagnosed cases have family history (mainly associated with C9ORF72 mutations [40\% of cases], superoxide dismutase-1 [SOD1] mutations [10-20\% of cases] and TAR DNA binding protein-43 [TDP-43, $4 \%$ of cases $]^{2}$ and remaining $90 \%$ of the cases are sporadic. The incidence of ALS has been reported to be 1.5 to 2.5 per 100,000 individuals per year ${ }^{3}$ with worldwide prevalence of 6 in 100,000 individuals. ${ }^{4}$ In India, approximately 5 of 100,000 individuals get affected from $\mathrm{ALS}^{5}$ with higher prevalence in males than females. ${ }^{6}$ Upper and lower motor neurons in the cerebral cortex, brainstem, and spinal cord degenerate because of which movement is affected. ${ }^{7}$ As the disease progresses, all voluntary muscles are affected and daily activities like walking, talking, eating are severely compromised. This is followed by adverse effect on involuntary muscles including respiratory, as well as cardiac muscles, proving to be life-threatening. Patients suffering from ALS die within 1 to 5 years of the detection of the disease because of respiratory or cardiac failure ${ }^{8}$ with a few patients surviving up to 10 years.

In spite of decades of research, the prognosis of the disease remains elusive with limited treatment strategies. Riluzole is the only Food and Drug Administration (FDA)-approved drug for ALS and can only provide symptomatic relief. ${ }^{9}$ Earlier diagnosis of the disease is challenging but critical for management of the ALS patients. Neuroinflammation is prominently correlated with ALS disease onset and is found to be associated with monocyte chemoattractant protein-1 (MCP-1), and other inflammatory cytokines (and receptors like CCR2), fibronectin, interleukins, etc. ${ }^{10} \mathrm{~A}$ marked variation in expression profile of these molecules has been described. In most of the familial ALS patients, at least one of these genes has been found to be affected. However, SOD1 is mutated in $20 \%$ of patients while TDP-43 mutation has been linked with 3 to $4 \%$ familial ALS cases. ${ }^{11}$ It has been shown that TDP-43 gets accumulated in the neurons of ALS patients, also termed as TDP-43 proteinopathy. Increased level of TDP-43 has been reported in the cerebrospinal fluid (CSF) and plasma of patients as compared with controls. ${ }^{12}$ Along with VEGF (vascular endothelial growth factor), VEGFR2 (VEGF receptor 2), and ANG (angiogenin; hypoxia responsive gene responsible for vascularization) are also believed to be associated with disease. Similarly, OPTN (optineurin) is known to be deposited as inclusion bodies but its levels in the plasma or serum not analyzed yet.

Protein expression analysis of various circulating proteins (in biofluid) has potential for biomarker discovery and can aid in the early diagnosis/prognosis and advancement in treatment strategies in ALS. For this reason, the expression of proteins known to be involved in this disease are routinely being examined in biological fluids and correlated with disease severity and progression. However, results from various studies lack consistency making their potential as a biomarker for disease prediction unreliable. For example, VEGF levels were found to be unaltered in ALS patients' CSF and spinal cord sections, ${ }^{13,14}$ while in the ALS serum, VEGF was reported to be elevated. ${ }^{15,16}$ We have earlier shown in a study on North Indian population that VEGF-A is increased in ALS patients' serum, as well as CSF. ${ }^{16}$ Similarly, studies related to other associated molecules in the ALS pathology, like ANG, ${ }^{17}$ TDP-43, ${ }^{18}$ MCP-1, have also showed diverse reports. Conclusively, ALS diagnostic efficacy can be enhanced by proposing a panel of protein expression chip to precisely identify the ALS with increasing efficacy.

Present study has attempted to examine the expression of these molecules including VEGF, ANG, TDP-43, OPTN, VEGFR2, and MCP-1 in ALS patient's plasma to propose the probable diagnostic panel for early diagnose ALS panel.

\section{Materials and Methods}

\section{Patient Recruitment and Sample Collection}

A total of 89 ALS patients and 98 genetically unrelated healthy controls were recruited for the study as per the informed consents, duly approved by the institutional ethical committee. Patients visiting outpatient department (OPD), who were clinically diagnosed to have ALS, were included. All the patients were found to have sporadic onset of disease without any family history. Mean ALS functional rating scale (FRS) score was found to be 34.59 . Patients were categorized according to the ALS FRS-R scoring into minimal, mild, and moderate-to-severe categories ${ }^{19}$ in accordance with increasing severity of the disease. Four criteria come under the ALS FRS-R covering functional assessment of trunk, cervical, lumbosacral region, and respiratory functions. Each section has three questions with answers ranging from $0-4$.

\section{Characteristics of the Patients Recruited: Sociodemographic Analysis}

Out of total 89 patients recruited, 67 participants were males and 22 were females, that is, nearly $75.28 \%$ of participants were males and $24.72 \%$ were females suggesting higher prevalence of the disease in males than females. The average age of all the ALS participants was 48.43 years and 41.98 years in case of controls. After measuring height and weight of the participants, the body mass index (BMI) was calculated as BMI $=$ weight $(\mathrm{kg}) /$ height $\left(\mathrm{m}^{2}\right)$. No significant difference was found between the BMI of patients (average, $22.7 \mathrm{~kg} / \mathrm{m}^{2}$ ) and control (average, $24.10 \mathrm{~kg} / \mathrm{m}^{2}$ ).

\section{Amyotrophic Lateral Sclerosis Diagnostic Classification}

The severity of ALS disease in Indian patients was based on ALS FRS R scoring. The ALS patients were classified into three categories as per the score obtained which includes minimal, mild, and moderate-to-severe. ALS FRS is the functional 
rating scale designed to assess the progression of the disease in ALS patients. The scale includes factors related to physical health and health of motor functions, as well as respiratory functions. Patients are categorized based on the scoring. ${ }^{20}$

El Escorial criteria is a diagnostic criteria for ALS patients. Patient group can be divided into the following three categories according to the criteria: definite, probable and possible. ${ }^{21}$

\section{Plasma Isolation}

Blood sample was taken in ethylene diamine tetra-acetic acid (EDTA) coated vials (BD vacutainers) and mixed thoroughly to avoid blood coating. The samples were layered on equal volume of Histopaque (HiSep LSM 1077, HiMedia Laboratories, Mumbai, Maharashtra, India) and then centrifuged at 1,500 rpm for 30.0 minutes at room temperature, kept at room temperature. Plasma was collected from upper layer which appeared as transparent fluid.

\section{Enzyme-Linked Immuno-sorbent Assay}

Sandwich enzyme-linked immunosorbent assay (ELISA) was used for the estimation of various molecules. Commercially available ELISA kits were used to estimate the protein levels for ANG, VEGF, VEGFR2, OPTN, TDP-43 (Qayee BioTechnology Co., Ltd., Shanghai, China), and MCP-1 (Diaclone SAS, Besancon, France) in plasma of participants as per the standard protocol described by manufacturer. Briefly, $50 \mu \mathrm{L}$ of standard and diluted samples (range: 2-10 times of dilution) were added to the wells, after which HRP conjugated antibody was added. The plate was set for incubation at $37^{\circ} \mathrm{C}$ for 1 hour. This allowed the antigen to bind with antibody precoated in the wells of ELISA plate. After washing the plate, $50 \mu \mathrm{L}$ of chromogen solution A and B were added in dark and the plate was incubated for 10 minutes at $37^{\circ} \mathrm{C}$ in dark. The stop solution was added and estimation was done using ELISA plate reader (iMARK reader, BioRad) at $450 \mathrm{~nm}$. OD values were noted.

\section{Total Protein Estimation}

Total protein was estimated by Bradford's method using Bovine serum albumin (BSA) as standard. Coomassie brilliant blue reagent (Bio-Rad Protein Assay Dye Reagent Concentrate, $450 \mathrm{~mL}$ no.: 5000006, Bio-Rad Laboratories, Hercules, California, United States) was used for total protein estimation and absorbance was taken at $595 \mathrm{~nm}$. ELISA values were normalized with total protein present in the sample. The mean and standard deviation was calculated for each protein.

\section{Statistical Analysis}

Data were analyzed by using SPSS version 21 . Data normality was analyzed using Kolmogorrov-Smirnov test and ShapiroWilk test depending upon the sample size. Mann-Whitney $U$-test was applied to compare ALS and normal control groups. Kruskal-Wallis test was employed to analyze the comparative protein levels in ALS subtypes including minimal, mild, and moderate-to-severe to see the protein variation with the disease severity of ALS, Spearman's analysis was done to see correlation between studied proteins and to see the probable mechanistic crosstalk in ALS pathology. Protein levels were also correlated with age, BMI, and duration of disease (in months) using Spearman's correlation analysis. The association of variations in the protein levels with various sociodemographic parameters was analyzed using parametric and nonparametric tests. The sociodemographic parameters included El Escorial criteria, gender, smoking habits, alcohol consumption, feeding habits, onset of the disease (early or late onset), and duration of the disease (short duration or long duration).

\section{Results}

\section{Protein Expressions in Indian Amyotrophic Lateral Sclerosis Patients \\ Differential Total Protein in Amyotrophic Lateral Sclerosis Patients}

Total protein estimation for the plasma samples of the patients and controls indicated increased level of the total protein concentration in the patient group as compared with controls ( $\boldsymbol{- \text { Fig. 1 }}$ ). These total protein values were used for normalization of the target protein concentrations estimated using ELISA.

\section{Target Protein Concentration}

ELISA results indicated lower levels of all the above-mentioned six proteins in ALS patients' plasma as compared with normal controls. OPTN levels were significantly reduced in patients ( - Fig. $2 \mathrm{~A}, p=6.9 \times 10^{-5}$ ). When categorized among minimum, mild, and moderate-to-severe, on the basis of disease progression, the protein levels were found to be marginally decreased in severe category compared with other subtypes. The decrease was, however insignificant ( - Fig. 3A, $p=0.443$ ). Plasma from ALS patients was found to have significantly lower levels of TDP-43 ( - Fig. 2B, $p=1.4 \times 10^{-3}$ ). Reduced expression both OPTN and TDP-43 are suggesting the proteinopathy in Indian ALS.

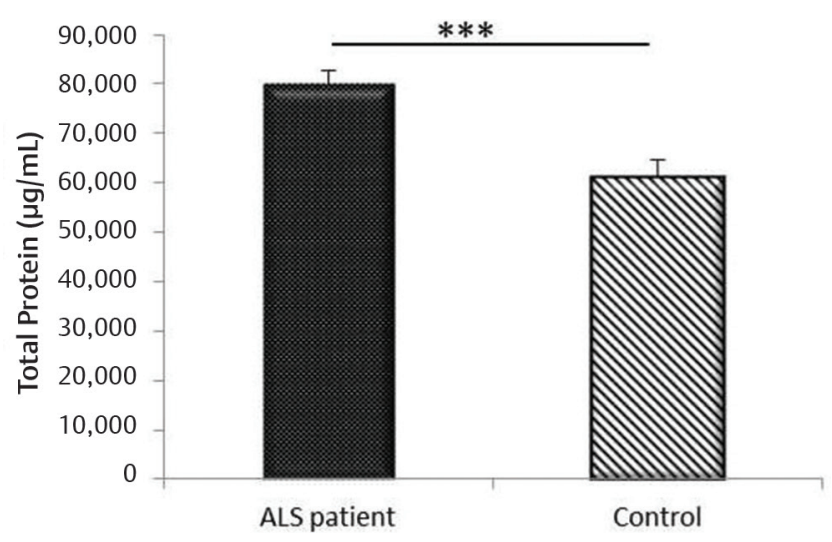

Fig. 1 Total protein expression and comparison of ALS patients and normal controls. ALS, amyotrophic lateral sclerosis. 

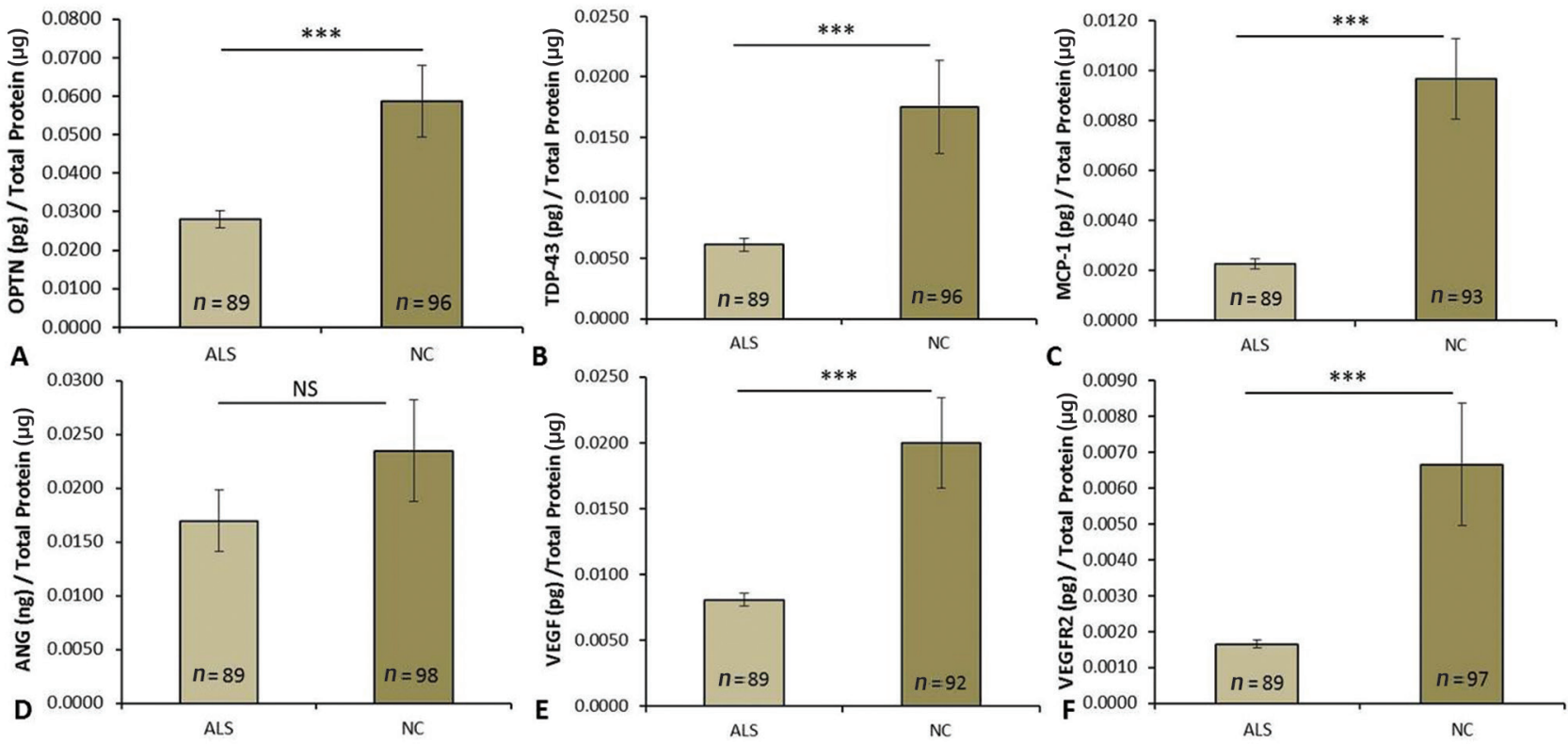

Fig. 2 Plasma protein levels of (A) optineurin (OPTN), (B) TDP-43 (TAR DNA binding protein), (C) MCP-1 (monocyte chemoattractant protein-1), (D) Angiogenin (ANG), (E) VEGF (vascular endothelial growth factor) and (F) VEGFR2 (VEGF receptor 2) estimated by ELISA in ALS patients and controls. All data are expressed as mean \pm SEM. Significance was considered at $p \leq 0.05,{ }^{* * *} p$ value $\leq 0.001$. ALS, amyotrophic lateral sclerosis; NC, normal control; NS, nonsignificant.

Moreover, significant decrease in level of MCP-1 was also reported in ALS patients compared with control ( - Fig. 2C, $\left.p<10^{-6}\right)$. Likewise, categorization according to ALSFRS score showed nonsignificant alterations in MCP-1 level with disease severity ( - Fig. 3B, $p=0.435$ ) suggesting that a minute changes in MCP-1 may ubiquitously stimulate ALS pathology.

When we analyzed the expression of angiogenic markers, for example, VEGF, VEGFR2, and ANG, we did not find any significant changes in ANG levels between ALS and controls ( - Fig. 2D, p = 0.262). Though, angiogenic proteins including VEGF ( - Fig. 2E, $p<10$ ) and VEGFR2 expressions ( - Fig. 2F, $p<3.4 \times 10$ ) were also significantly decreased in ALS patients in comparison to controls. However, similar to other proteins, the comparison of protein levels among the subcategories of ALS patients did not reveal any marked difference. Though downward trend was observed for VEGF ( $~-$ Fig. 3C, $p=0.335$ ), ANG ( - Fig. 3D, $p=0.703$ ), TDP-43 ( - Fig. 3E, $p=0.638$ ), and VEGFR2 ( - Fig. 3F, $p=0.808$ ) with increased disease severity yet the difference was insignificant.

\section{Protein-Protein Correlation}

ELISA results have showed the decreased expression of studied proteins. However, biological interactions between them to show pathological significance in ALS have not been established yet. We have analyzed the multiple correlation using Spearman's test to investigate the protein-protein interactions, as well as correlation of protein levels, with age, BMI, and duration of disease in Indian ALS patients. Results have showed strong positive correlation between all studied proteins. These proteins may be interdependent (-Table $\mathbf{1}$ ). The pathological characteristics has also suggested the neuroinflammatory-, angiogenic-, and proteinopathy-associated changes in ALS patients. Results implicate the prospective interactions and cross-talk between these proteins in the progression of ALS pathology in Indian population. No significant correlation was found between protein levels and parameters such as age, BMI, and duration of disease (-Supplementary Table 1; available online only). Interestingly, results have revealed significant alter levels of ANG in definite, probable, and possible ALS patients based on EI Escorial scoring (-Supplementary Table 2; available online only).

\section{Discussion}

Analysis of biomarkers can be useful in the early diagnosis of diseases. Especially having panel of molecules can help better in diagnosis of disease, instead of analyzing a single molecule in plasma. Having chip-based tools that can analyze panel of interacting biomarkers for a disease can prove helpful in diagnosis, as well as prognosis of disease. Plasma proteomics is being used increasingly for the analysis of concentrations of various biomarkers in the blood, although it is very expensive. Plasma is considered as an important biofluid for assessing diffusion of proteins from several tissues. Current research is directed in strengthening early diagnosis of ALS or at least analyzing the prognosis of disease via varying protein levels. VEGF is a major angiogenic molecule that is responsible for vascularization at the time of development, as well as later in life. Various studies have assessed the concentration of VEGF in serum, plasma, and CSF of ALS patients at various stages of the disease. VEGF levels in serum and CSF have been found to be increased in some of the studies. For example, Gao et al also measured VEGF concentration in patients at 

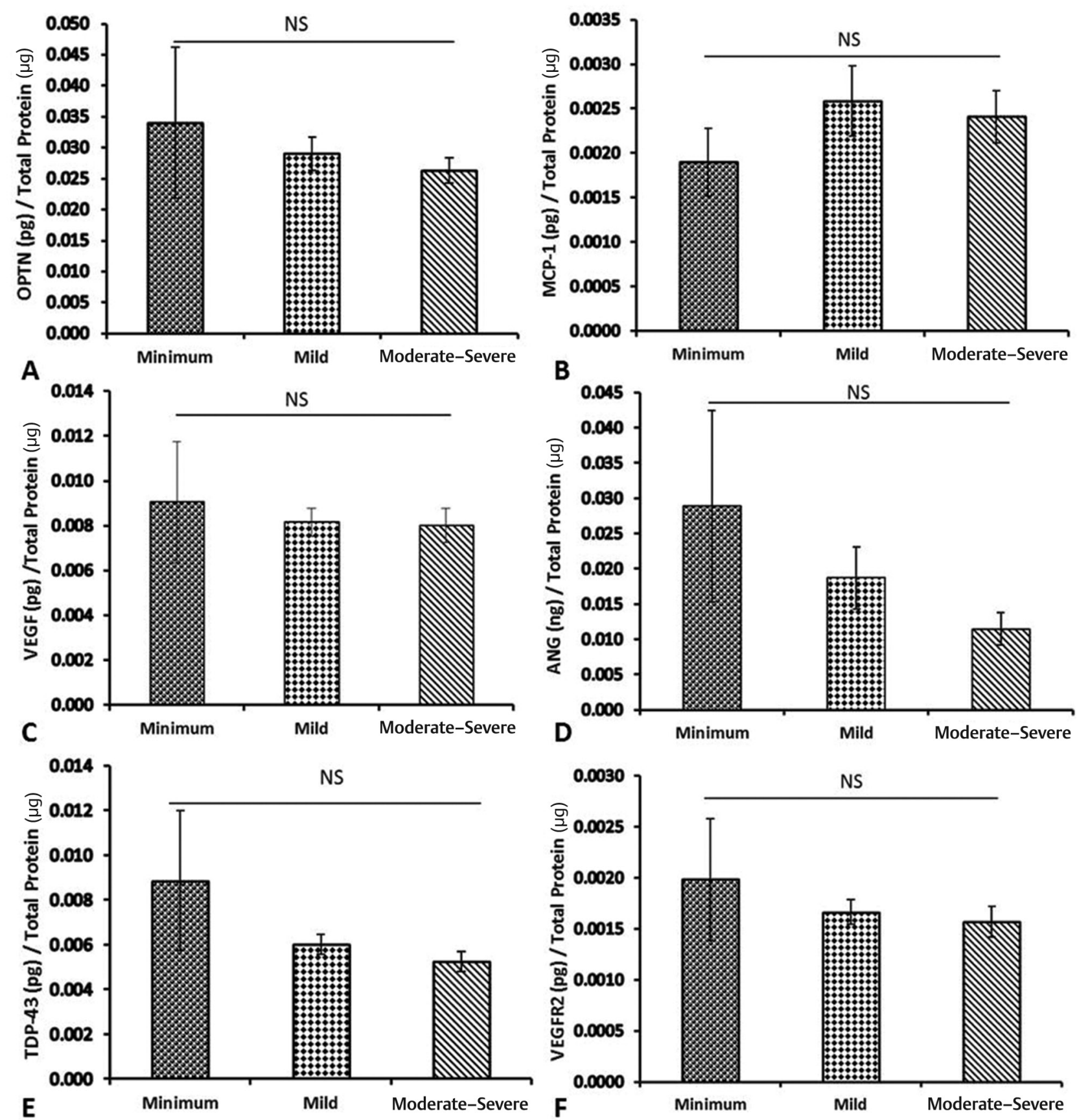

Fig. 3 Association of severity of ALS (minimum, mild, and moderate-severe) based on ALS FRS-R score with (A) optineurin (OPTN), (B) MCP-1 (monocyte chemoattractant protein-1) (C) VEGF (vascular endothelial growth factor), (D) angiogenin (ANG), (E) TDP-43 (TAR DNA binding protein) and (F) VEGFR2 (VEGF receptor 2) in ALS patients. Data are represented as mean with standard error as error bar. Significance was calculated by Kruskal-Wallis test and considered at $p \leq 0.05$. ALS, amyotrophic lateral sclerosis; FRS, functional ration scale; NS, nonsignificant.

different time intervals after the disease onset. According to this study, the upregulation of VEGF level was found to be more in patients with the progression of the disease rather than during 12 months. ${ }^{15}$ They argued that VEGF levels get upregulated in patients as the disease progresses. ${ }^{16}$ However, Nygren et al showed that CSF concentration of VEGF is not increased in patients. ${ }^{14}$ When the postmortem spinal cord sections were analyzed, it was similarly found that there was no increase in VEGF levels. But in our study, we found opposite trend in Indian patients. VEGF level was decreased significantly in patients as compared with controls, although we did not carry out the autopsy studies. More research in this direction can clarify this variation depending upon family history and demographic analysis. As VEGF is required for angiogenesis, decreased VEGF concentration may exacerbate degeneration of motor neurons because of hypoxia created by reduced vascularization. ${ }^{22,23}$ This can also explain the increase in ANG levels of patients. VEGF levels reported 
Table 1 Spearman's correlation analysis between studied proteins in Indian ALS pathology

\begin{tabular}{|l|l|l|l|l|l|l|}
\hline MCP-1 & $r=1$ & & & \\
\hline VEGF & $\begin{array}{l}r=0.599 \\
p<10^{-6}\end{array}$ & $r=1$ & & \\
\hline VEGFR2 & $\begin{array}{l}r=0.591 \\
p<10^{-6}\end{array}$ & $\begin{array}{l}r=0.596 \\
p<10^{-6}\end{array}$ & $r=1$ & & \\
\hline ANG & $\begin{array}{l}r=0.639 \\
p<10^{-6}\end{array}$ & $\begin{array}{l}r=0.411 \\
p=6.4 \times 10^{-5}\end{array}$ & $\begin{array}{l}r=0.772 \\
p<10^{-6}\end{array}$ & $r=1$ & \\
\hline TDP-43 & $\begin{array}{l}r=0.583 \\
p<10^{-6}\end{array}$ & $\begin{array}{l}r=0.446 \\
p=1.2 \times 10^{-5}\end{array}$ & $\begin{array}{l}r=0.759 \\
p<10^{-6}\end{array}$ & $\begin{array}{l}r=0.833 \\
p<10^{-6}\end{array}$ & $r=1$ & \\
\hline OPTN & $\begin{array}{l}r=0.723 \\
p<10^{-6}\end{array}$ & $\begin{array}{l}r=0.888 \\
p<10^{-6}\end{array}$ & $\begin{array}{l}r=0.792 \\
p<10^{-6}\end{array}$ & $\begin{array}{l}r=0.662 \\
p<10^{-6}\end{array}$ & $\begin{array}{l}r=0.620 \\
p<10^{-6}\end{array}$ & $r=1$ \\
\hline $\begin{array}{l}\text { Spearman's } \\
\text { Correlations }\end{array}$ & $\mathbf{M C P}-1$ & VEGF & VEGFR2 & ANG & TDP43 & OPTN \\
\hline
\end{tabular}

Abbreviations: ANG, angiogenin; ALS, amyotrophic lateral sclerosis; MCP-1, monocyte chemoattractant protein-1; OPTN, optineurin; TDP, TAR DNA binding protein; VEGF, vascular endothelial growth factor; VEGFR2, VEGF receptor 2.

in various studies have shown mixed results. ${ }^{17}$ In our study, VEGF was found decreased. It can be hypothesized that decreased VEGF levels might lead to hypoxia in response to which hypoxia inducing factor (HIF- $\alpha$ ) getting activated that may further induce ANG expression, either as a compensatory response to hypoxia associated with low-VEGF levels or exerting neurogenic effects. Although, our study didn't show any significant difference of ANG in patients as compared with controls, Cronin et al reported increased ANG expression. In this context, it is pertinent to state that Cronin et al did not report any correlation between VEGF and ANG levels. ${ }^{17}$ But in our study, we found significant correlation between both the molecules.

In our study,TDP-43 levels were found decreased significantly in ALS patients when analyzed by ELISA.TDP-43 proteinopathy is an important characteristic of ALS and frontotemporal dementia (FTD). ${ }^{24,25}$ Granules of TDP-43 are found to get aggregated in the cytoplasm of neurons ${ }^{26}$ due to which neurons begin to degenerate. Expectedly, TDP-43 concentration in plasma and serum has been reported to be increased in several studies, ${ }^{12,27}$ albeit no such study from India has so far analyzed this. TDP-43 is involved in the regulation of angiogenic genes. ${ }^{28}$ TDP-43 regulates progranulin in tandem with VEGF. Briefly, it acts as an inducer of angiogenic genes which can be studied in tandem while analyzing TDP-43 role in ALS. Further, TDP-43 proteins have a nuclear localization signal that allows it to enter the nucleus and act as inducer. However, in the various cases of ALS, disruption of nuclear localization signal (NLS) causes formation of TDP-35 and TDP-25 fragments which start accumulating in cells and form protein aggregates. These protein aggregates further entrap TDP-43 molecules and form protein inclusions. ${ }^{29}$ Therefore, it is attractive to hypothesize that hypoxia, being an important risk factor in ALS, coupled with importance of angiogenesis in the neuroprotection, TDP-43 levels might influence the angiogenic pathway in severe forms of ALS. However, this needs to be examined in larger sample size.

In developing an understanding of angiogenesis-hypoxia cross talk in ALS, VEGFR2'srole in ALS cannot be underestimated, as it acts as a receptor of VEGF. In our study, its levels showed decreasing trends. It is pertinent to point out that there is decrease in VEGFR2/VEGFR1 as reported in various studies. ${ }^{30,31}$ Decrease in VEGFR2 can be ascribed to feedback loop moderated by VEGF expression. The discussion of angiogenesis-hypoxia axis in the pathogenesis of ALS is incomplete without reviewing the cross talk of OPTN with TDP-43. OPTN has nuclear factor-KB (NF-KB) suppressive activity and inhibits the tumor necrosis factor (TNF)- $\alpha$-mediated NF-кB activation. Mutations in the OPTN activate TNF- $\alpha$ and the caspase pathway, ${ }^{32,33}$ disrupting the nuclear localization signal of TDP-43. Consequently, it is found increased and accumulated as protein inclusions in the motor neurons of ALS patients as shown by IHC studies on post mortem spinal cord sections. ${ }^{34}$ For decrease in TDP43 values, as found in our study, it can be hypothesized that excessive accumulation of TDP43 in cells can be a cause of decreased plasma levels of TDP-43. As TDP-43 is unable to target the nucleus, it cannot induce VEGF- (angiogenic genes) causing hypoxia. In this study, we found a decrease in OPTN in plasma of ALS patients, possibly hampering the regulation of TDP-43, as postulated above.

MCP-1 was also found to be significantly decreased in ALS patients. MCP-1 levels are also known to be elevated in ALS patients due to associated neuroinflammation in the progression of ALS. ${ }^{16}$ As the severity of the disease progresses, the alterations are expected to be enhanced. OPTN, VEGF, ANG, TDP-43, and VEGFR2 show decreasing trend as the severity of the disease progresses (in accordance with ALSFRS scoring) but the difference was not significant. Along with discussing differential levels of various proteins, we tried to hypothesize the possible interaction pattern between these proteins and that such panels of interacting molecules can be studied for analyzing their diagnostic or prognostic potential. Possible association and interaction between these molecules have been presented in $\boldsymbol{- F i g} . \mathbf{4}$.

\section{Conclusion}

The candidate biomarkers analyzed in this study showed fluctuating trends in the plasma of ALS patients. VEGF, VEGFR2, OPTN, TDP-43, and MCP-1 were downregulated and were 


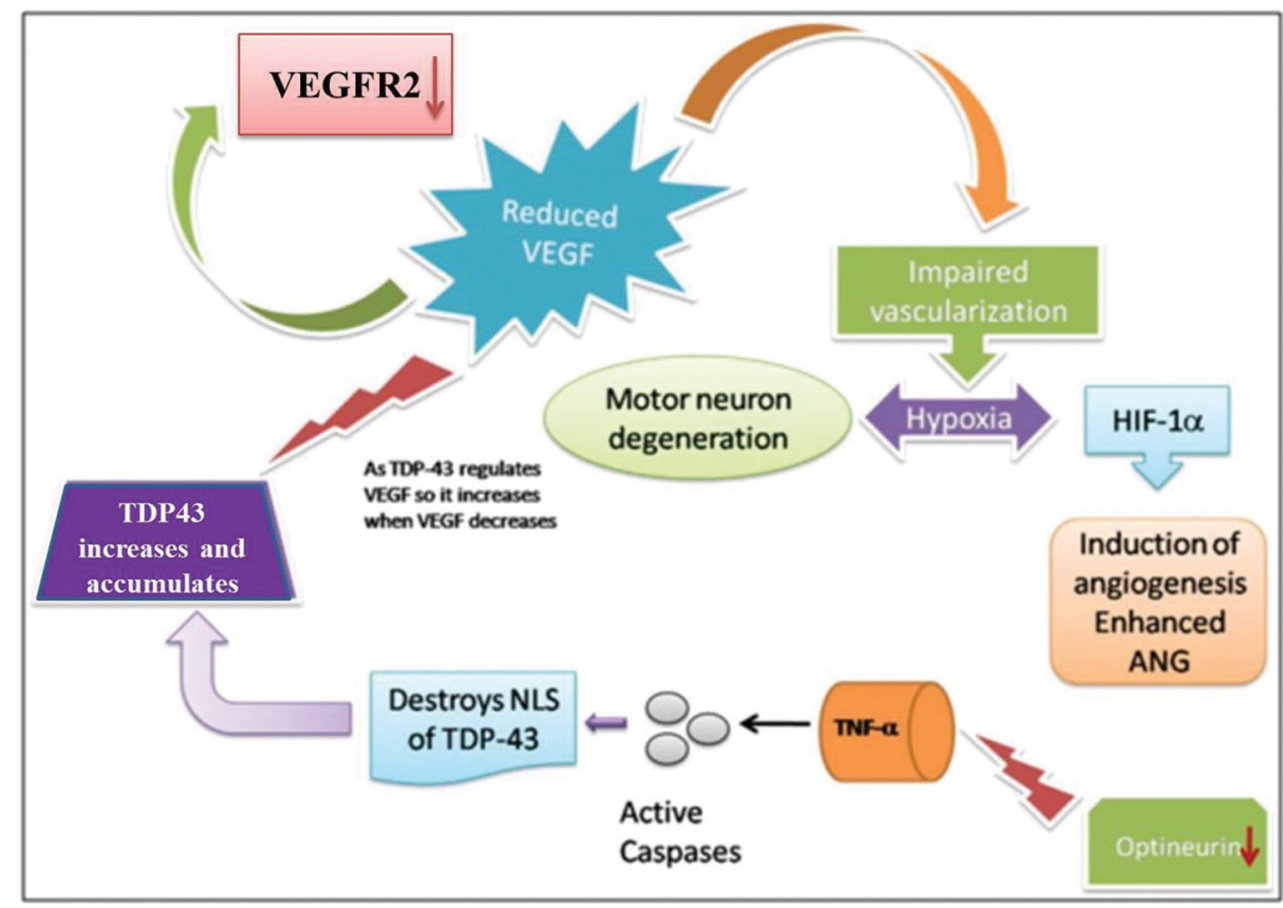

Fig. 4 Proposed schematic showing the role of six major proteins in causing ALS-reduced VEGF may be responsible for the hypoxia in brain because of which HIF-1 $\alpha$ (hypoxia inducing factor- $1 \alpha$ ) gets activated consequently inducing ANG activation to compensate hypoxia. Because VEGF is reduced, its receptor soluble VEGFR2 may also be downregulated. Decreased VEGF may also lead to enhanced TDP-43 which may compensate for reduced VEGF by increasing its expression. Decreased OPTN also leads to disruption of NLS (nuclear localization signal) of TDP43 due to which it gets accumulated in the cytoplasm. ALS, amyotrophic lateral sclerosis; ANG, angiogenin; OPTN, optineurin; TDP, TAR DNA binding protein; VEGF, vascular endothelial growth factor; VEGFR2, VEGF receptor 2.

positively correlated to each other, suggesting a cross-talk exists among these five biomarkers. A comprehensive study is required to analyze the effect of these biomarkers on the disease progression to understand the role in the disease progression or for early diagnosis of the disease. Further research in this direction is required.

\section{Note}

Each demographic factor is divided into categories and the mean protein levels of markers were compared for the respective categories. No significant difference was found between protein levels with respect to various categories of these factors

\section{Authors' Contributions}

S.M.: planning and execution of experiments, data generation and analysis, writing, and editing of manuscript; R.K.: experimentation, data generation and analysis, writing, and editing of manuscript; A.T.: experimentation and data generation. K.S.: data analysis and editing of manuscript; A.A.: corresponding author and editing of manuscript.

\section{Funding}

The study was funded by ICMR (Indian council of Medical Research), UGC-fellowship.

\section{Acknowledgement}

The authors sincerely acknowledge Keshav Thakur and Pawan Gupta for sample collection and Dr. S. Prabhakar for the screening of patients. They also acknowledge Professor
Kamal Kishore, Department of Biostatistics, PGIMER, Chandigarh, for the statistical analysis of the data.

\section{Conflict of Interest}

None declared.

\section{References}

1 Boillée S, Vande Velde C, Cleveland DW. ALS: a disease of motor neurons and their nonneuronal neighbors. Neuron 2006;52(1):39-59

2 Renton AE, Chiò A, Traynor BJ. State of play in amyotrophic lateral sclerosis genetics. Nat Neurosci 2014;17(1):17-23

3 Logroscino G, Traynor BJ, Hardiman O, et al; EURALS. Descriptive epidemiology of amyotrophic lateral sclerosis: new evidence and unsolved issues. J Neurol Neurosurg Psychiatry 2008;79(1):6-11

4 Talbott EO, Malek AM, Lacomis, M D The epidemiology of amyotrophic lateral sclerosis. In: Aminoff MJ, Boller F, Swaab DF, eds. Handbook of Clinical Neurology. Vol. 138. Amsterdam, The Netherlands: Elsevier; 2016 225-238

5 Raj P. Amyotrophic Lateral Sclerosis. Rare Diseases India. Available at: http://www.rarediseasesindia.org/. Accessed June 11, 2020

6 Manjaly ZR, Scott KM, Abhinav K, et al. The sex ratio in amyotrophic lateral sclerosis: a population based study. Amyotroph Lateral Scler 2010;11(5):439-442

7 Zarei S, Carr K, Reiley L, et al. A comprehensive review of amyotrophic lateral sclerosis. Surg Neurol Int 2015;6:171

8 Corcia P, Pradat PF, Salachas F, et al. Causes of death in a post-mortem series of ALS patients. Amyotroph Lateral Scler 2008;9(1):59-62 
9 Borrás-Blasco J, Plaza-Macías I, Navarro-Ruiz A, Perís-Martí J, Antón-Cano A. [Riluzole as a treatment for amyotrophic lateral sclerosis] (in Spanish) Rev Neurol 1998;27(160):1021-1027

10 Turner MR, Kiernan MC, Leigh PN, Talbot K. Biomarkers in amyotrophic lateral sclerosis. Lancet Neurol 2009;8(1):94-109

11 Liscic RM, Breljak D. Molecular basis of amyotrophic lateral sclerosis. Prog Neuropsychopharmacol Biol Psychiatry 2011;35(2):370-372

12 Verstraete E, Kuiperij HB, van Blitterswijk MM, et al. TDP43 plasma levels are higher in amyotrophic lateral sclerosis. Amyotroph Lateral Scler 2012;13(5):446-451

13 Iłzecka J. Cerebrospinal fluid vascular endothelial growth factor in patients with amyotrophic lateral sclerosis. Clin Neurol Neurosurg 2004;106(4):289-293

14 Nygren I, Larsson A, Johansson A, Askmark H. VEGF is increased in serum but not in spinal cord from patients with amyotrophic lateral sclerosis. Neuroreport 2002;13(17):2199-2201

15 Gao L,Zhou S, Cai H, Gong Z,Zang D. VEGFlevels in CSF and serum in mild ALS patients. J Neurol Sci 2014;346(1-2):216-220

16 Gupta PK, Prabhakar S, Sharma S, Anand A. Vascular endothelial growth factor-A (VEGF-A) and chemokine ligand-2 (CCL2) in amyotrophic lateral sclerosis (ALS) patients. J Neuroinflammation 2011;8(1):47

17 Cronin S, Greenway MJ, Ennis S, et al. Elevated serum angiogenin levels in ALS. Neurology 2006;67(10):1833-1836

18 Chou C-C, Zhang Y, Umoh ME, et al. TDP-43 pathology disrupts nuclear pore complexes and nucleocytoplasmic transport in ALS/FTD. Nat Neurosci 2018;21(2):228-239

19 Armon C. How are ALS Functional Rating Scale (ALSFRS) scores interpreted in the assessment of amyotrophic lateral sclerosis (ALS)? Available at: https://www.medscape.com/ answers/1170097-81928/81928-print. Accessed June 11, 2020

20 Cedarbaum JM, Stambler N, Malta E, et al; BDNF ALS Study Group (Phase III). The ALSFRS-R: a revised ALS functional rating scale that incorporates assessments of respiratory function. J Neurol Sci 1999;169(1,2):13-21

21 Agosta F, Al-Chalabi A, Filippi M, et al; WFN Research Group on ALS/MND. The El Escorial criteria: strengths and weaknesses. Amyotroph Lateral Scler Frontotemporal Degener $2015 ; 16(1,2): 1-7$

22 Oosthuyse B, Moons L, Storkebaum E, et al. Deletion of the hypoxia-response element in the vascular endothelial growth factor promoter causes motor neuron degeneration. Nat Genet $2001 ; 28(2): 131-138$
23 Lambrechts D, Storkebaum E, Morimoto M, et al. VEGF is a modifier of amyotrophic lateral sclerosis in mice and humans and protects motoneurons against ischemic death. Nat Genet 2003;34(4):383-394

24 Mackenzie IR, Neumann M, Bigio EH, et al. Nomenclature and nosology for neuropathologic subtypes of frontotemporal lobar degeneration: an update. Acta Neuropathol 2010;119(1):1-4

25 Liscic RM, Grinberg LT, Zidar J, Gitcho MA, Cairns NJ. ALS and FTLD: two faces of TDP-43 proteinopathy. Eur J Neurol 2008;15(8):772-780

26 Kwong LK, Neumann M, Sampathu DM, Lee VM-Y, Trojanowski JQ. TDP-43 proteinopathy: the neuropathology underlying major forms of sporadic and familial frontotemporal lobar degeneration and motor neuron disease. Acta Neuropathol 2007;114(1):63-70

27 Feneberg E, Gray E, Ansorge O, Talbot K, Turner MR. Towards a TDP-43-based biomarker for ALS and FTLD. Mol Neurobiol 2018;55(10):7789-7801

28 Colombrita C, Onesto E, Megiorni F, et al. TDP-43 and FUS RNA-binding proteins bind distinct sets of cytoplasmic messenger RNAs and differently regulate their post-transcriptional fate in motoneuron-like cells. J Biol Chem 2012;287(19):15635-15647

29 Cicardi ME, Cristofani R, Rusmini P, et al. Tdp-25 Routing to Autophagy and Proteasome Ameliorates its Aggregation in Amyotrophic Lateral Sclerosis Target Cells. Sci Rep 2018;8(1):12390

30 Brockington A, Wharton SB, Fernando M, et al. Expression of vascular endothelial growth factor and its receptors in the central nervous system in amyotrophic lateral sclerosis. J Neuropathol Exp Neurol 2006;65(1):26-36

31 Anand A, Gupta PK, Sharma NK, Prabhakar S. Soluble VEGFR1 (sVEGFR1) as a novel marker of amyotrophic lateral sclerosis (ALS) in the North Indian ALS patients. Eur J Neurol 2012;19(5):788-792

32 Nakazawa $\mathrm{S}$, Oikawa $\mathrm{D}$, Ishii $\mathrm{R}$, et al. Linear ubiquitination is involved in the pathogenesis of optineurin-associated amyotrophic lateral sclerosis. Nat Commun 2016;7(1):12547

33 Slowicka K, van Loo G. Optineurin functions for optimal immunity. Front Immunol 2018;9:769

34 Arai T, Hasegawa M, Akiyama H, et al. TDP-43 is a component of ubiquitin-positive tau-negative inclusions in frontotemporal lobar degeneration and amyotrophic lateral sclerosis. Biochem Biophys Res Commun 2006;351(3):602-611 